\title{
Efficient Route Discovery Using Channel Adaptive Probabilistic Broadcasting in Zigbee Wireless Sensor Networks
}

\author{
Haitham Y. Adarbah \\ Faculty of Foundation Studies \\ Gulf College \\ Muscat, Oman \\ Haitham.adarbah@gulfcollege.edu.om
}

\author{
Shakeel Ahmad \\ School of Media Arts \& Technology \\ Southampton Solent University \\ Southampton, United Kingdom \\ Shakeel.Ahmad@solent.ac.uk
}

\begin{abstract}
Low-Rate Wireless Sensor Networks (LR-WSNs) has become an active area of research in recent decades. ZigBee is a wireless standard based on IEEE 802.15.4 for LR-WSNs. Since in Zigbee networks energy is one of the most vital issues, the routing protocols must be energy efficient. By default, ZigBee uses an Ad hoc On-Demand Distance Vector (AODV) routing protocol. In AODV, broadcasting is the backbone of the route discovery process. Pure flooding is the simplest and most common broadcasting technique for route discovery in AODV routing. The obvious drawback of pure flooding is excessive redundant traffic that degrades the system performance. This is commonly known as broadcast storm problem (BSP). Several schemes have been proposed to address BSP, one of them is the Channel Adaptive Probabilistic Broadcast (CAPB) scheme that adapts the rebroadcast probability dynamically to the current Signal to Interference plus Noise Ratio (SINR) and node density in the neighbourhood. This paper evaluates the CAPB scheme in LRWSNs based on the IEEE 802.15.4/ZigBee. The CAPB scheme is implemented in the standard AODV routing protocol to replace the pure flooding based broadcast. Extensive ns-2 simulation results show that the CAPB scheme outperforms the standard AODV and the fixed probabilistic broadcasting scheme significantly in terms of routing overhead, throughput, end-to-end delay and energy consumption in noisy LR-WSNs.
\end{abstract}

Keywords- ZigBee; Low Rate Wireless Sensor Network; IEEE 802.15.4 MAC; Route Discovery; Channel Adaptive Probabilistic Broadcast; Broadcast Storm Problem; Probabilistic scheme.

\section{I.INTRODUCTION}

The proliferation of applications based on IEEE 802.15.4/Zigbee (e.g., monitoring physical or environmental conditions, such as temperature, sound, vibration etc.) have kept LR-WSNs an active area of research over the past two decades [1]. An LR-WSN is a self-configuring, self-healing and infrastructure-less network of mobile nodes connected to each other over single-hop or multi-hop wireless links on the ad-hoc basis [2].

ZigBee is a wireless standard based on IEEE 802.15.4 standard for PAN (Personal Area Networks) [3]. Its network and application layers are defined on the top of physical and data link layers standardized in IEEE 802.15.4. ZigBee networks use AODV (Ad-hoc On Demand Distance Vector) routing protocol by default [4].
ZigBee was designed to provide cost effective and energy efficient wireless communication networks. This makes ZigBee networks an ideal choice for a number of applications e.g., consumer electronics, industrial controls, PC peripherals, toys and games, and quick deployment of networks without requiring huge infrastructure.

In mobile LR-WSNs, nodes can be located arbitrarily within an area and are free to move. The movement of LR-WSN nodes changes the network topology dynamically. Mobile LR-WSN nodes adapt to the changing topology by discovering new neighbours and establishing new routes to destination nodes [5]. A node may not communicate directly with a distant node due to limited transmission range and may have to rely on other nodes to relay the message along the route to the final destination node. In this way, each node acts as a host node as well as a relay node to extend the reachability of other nodes.

When a node wants to send data to a remote node, first, it finds out a set of relay nodes between itself and the remote node. The process of finding the optimal set of relay nodes between the source node and the destination node is called route discovery. Node mobility, limited battery power and the errorprone nature of wireless links are the main challenges in designing an efficient route discovery process in LR-WSNs.

In AODV routing protocol, the routing process consists of two phases namely route-discovery and route-maintenance. These protocols rely on broadcasting for route discovery. For example, a source node that needs to send data to a destination node triggers route discovery mechanism by broadcasting a special control packet, called Route Request (RREQ), to its neighbours who then rebroadcast the RREQ packet to their neighbours. The process continues until the RREQ packet arrives at the destination node. The destination node sends a control packet called Route Reply (RREP) that follows the path of RREQ in reverse direction and informs the source node that a route has been established. Since every node on receiving the RREQ for the first time rebroadcasts it, it requires T-2 rebroadcasts in a network of T nodes assuming the destination is reachable. More details can be found in [7]. 


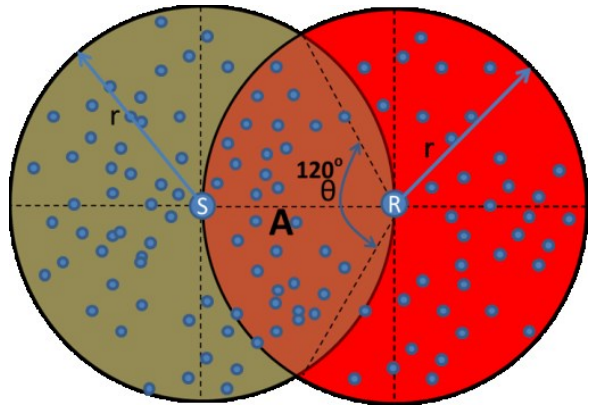

Fig. 1. Node R receives RREQ from node S [7].

Pure flooding often results in substantial redundant transmissions because a node may receive the same packet from multiple other nodes. This phenomenon, commonly known as the broadcast storm problem (BSP) [7], leads to increased communication overhead and serious performance complications in densely populated WSNs. The BSP equally affects the route maintenance phase during which routes are refreshed by triggering new route discovery requests to replace the broken routes. To address the BSP, the authors have previously proposed CAPB (Channel Adaptive Probabilistic Broadcasting) scheme for mobile ad-hoc networks [6]. The CAPB scheme adapts the broadcasting scheme dynamically according to the thermal noise, co-channel interference and node density in the neighbourhood.

This paper evaluates the performance of the CAPB in LRWSN based on IEEE802.15.4/Zigbee. The CAPB scheme is implemented in the network simulator ns-2 and its performance has been compared with the State of Art (SoA) schemes in terms of routing overhead, throughput, end-to-end delay and energy consumption. The simulation results showed that the proposed scheme outperforms the SoA broadcast schemes significantly.

The rest of the paper is organised as follows: Section 2 reviews ZigBee and IEEE 802.15.4. Section 3 presents the related work, Section 4 presents the CAPB broadcast scheme, and Section 5 presents simulation results and analysis followed by conclusions in Section 6.

\section{II.OVERVIEW OF ZIGBEE AND IEEE 802.15.4}

The ZigBee Alliance defines application, security and network layers for IEEE 802.15.4 devices [3]. Mainly, the network layer includes mechanisms for network topology setup and routing issues, whereas the application and security layers provide a framework for application support and security services.

The ZigBee network layer supports three types of topologies namely star, tree and mesh. In a Star topology, a node (or device) transmits packets directly to the ZigBee coordinator, and nodes do not communicate directly. In tree topology, the ZigBee coordinator creates a tree routed based on the MAC parent-child relationships between IEEE 802.15.4 devices. In a Mesh topology, non-parent-child devices can transmit directly to each other. In this situation, a reactive routing protocol like AODV is used. Even though in mesh topologies the parent-child tree structure is always created by the IEEE 802.15.4 MAC layer to start a new PAN, a hierarchical tree structure is not used for routing purposes.

An IEEE 802.15.4 WPAN (Wireless PAN) consists of a set of nodes and a PAN coordinator [8]. The network is controlled by the PAN coordinator which initiates network operations. Association procedure and realignment procedure are the procedures to establish and maintain device membership in a WPAN [1]. The PAN coordinator defines a set of procedures in order to build a new WPAN, and mechanism allowing new nodes to join the WPAN. A node can join WPAN using the association procedure which consists of three step: 1) scan the availability of WPANs; 2) select the WPAN; 3) start the association procedure with the PAN coordinator or another device that already has joined the selected WPAN. Once a node is connected to a WPAN, nodes maintain it and re-associate (if required) by using realignment procedure.

\section{III.RELATED WORK}

A number of research works have addressed the performance evaluation of routing protocols in IEEE 802.15.4/Zigbee networks $[9,10,11]$.

Nefzi and Song [10] analyzed ZigBee routing protocols in terms of delay performance and energy consumption. Their simulation results showed that Hierarchical Tree Routing provided good average end to end delay; however, performed poorly in terms of energy consumption.

Ortiz et al [12] proposed a novel fuzzy-logic based metric to be used in the decision-making process of AODV. In their suggestion, the metric evaluated important node features during route creation and selects, hop by hop, the best option in order to obtain energy and delay efficient routes. Their simulation results revealed that the proposed scheme showed a reduction in the communication delay, number of packets and overhead, improving route efficiency and reducing packet overload.

Peng et. al [13] presented an energy-aware routing mechanism EA-AODV to enhance the usage of energy and prolong the lifetime of ZigBee network. The simulation results of [13] showed that the EA-AODV was working well for saving energy and could improve the ZigBee network performance.

To the best of the authors' knowledge, no previous work has considered the effects of thermal noise, co-channel interference, and neighbourhood node density in IEEE 802.15.4/Zigbee networks to optimise the route discovery in AODV protocol.

\section{IV.THE CAPB ALGORITHM}

This section briefly describes the Channel Adaptive Probabilistic Broadcast (CAPB) scheme that adjusts the probability of rebroadcasting RREQ packets dynamically according to the SINR (Signal to Interference plus Noise Ratio) and node density in the neighbourhood.

The CAPB scheme works as follow: when node $\mathrm{R}$ receives an RREQ packet, for which $\mathrm{R}$ is not the destination node, it rebroadcasts the RREQ packet with probability $P_{\text {reb }}$. To determine the value of $P_{r e b}$, node R first determines the value 
of $N_{\text {eff }}$ which is the number of effective nodes within its transmission range $r$ which might have received the same RREQ packet. This is done as follows. We assume $N$ is the total number of nodes within the transmission range of node $\mathrm{R}$. The value of $N$ is inferred using Hello Packets. The nodes which are located within the transmission range of both nodes $\mathrm{R}$ and node $\mathrm{S}$ are shown by the overlapped area $\mathbf{A}$ of the two circles in Figure 1 (details can be found in [7]). If PER is the packet loss rate, using geometry it can be given as follow:

$$
N_{e f f}=N \times\left(\frac{\theta}{180}-\frac{\sin \theta}{\pi}\right)(1-P E R)
$$

A higher value of $N_{e f f}$ implies that more nodes have received the RREQ and consequently the value of $P_{r e b}$ should be lower and vice versa. This suggests an inverse relationship between $P_{\text {reb }}$ and $N_{\text {eff }}$.

$$
P_{r e b}=d \times \frac{1}{N_{e f f}}
$$

Here $d$ is a constant value representing the dissemination factor. The value of $d$ is greater than unity to compensate the $P E R$. For very low $\left(\leq N_{l}\right)$ and very high $\left(\geq N_{u}\right)$ values of $N_{\text {eff }}$ equation (2) may not hold true so fixed values of $P_{r e b}$ are used instead. The values of $N_{l}$ and $N_{u}$ can be derived from an estimated maximum and minimum possible node density and the transmission range of nodes. In general $P_{r e b}$ can be given as follows:

$$
P_{r e b}=\left\{\begin{array}{lr}
P_{\text {max }}, & \text { for } N_{\text {eff }} \leq N_{l} \\
d \times \frac{1}{N_{e f f}}, & \text { for } N_{l}<N_{e f f}<N_{u} \\
P_{\text {min }}, & \text { for } N_{\text {eff }} \geq N_{u}
\end{array}\right.
$$

\section{V.Performance EVALUATION Of THE CAPB Scheme}

\section{A. Simulation setup}

We used ns-2 simulator $(2.35 \mathrm{v})$ to implement and evaluate the CAPB scheme in LR-WSNs using AODV routing protocol. Standard AODV uses pure flooding. The CAPB and a probabilistic broadcasting scheme (AODV-P) have been implemented in the route discovery process of AODV to replace pure flooding. In AODV-P scheme, $\mathrm{P}$ shows the probability of rebroadcasting and is set to 0.6 after running the simulation with a range of values for $\mathrm{P}$ and choosing the one giving the best performance. The radio propagation is based on 2-ray Ground Reflected Model. The network bandwidth is set to $250 \mathrm{kbps}$, medium access control (IEEE 802.15.4 MAC) protocol is simulated using the ns2 library [14], and interface queue is set to 100. The node mobility is modelled using Random Waypoint mobility model with variable node speed [15] and pause time is set to zero. The scenario consists of 400 nodes which are placed randomly in an area of 200x200 meters. Only the sink node (that represents the coordinator node) is deployed in the centre of the area [coordinates: 100,100]. Transmission power, path loss and receive power threshold are set such that the effective transmission range is $10 \mathrm{~m}$ to suit LR-WSNs. Each node uses UDP protocol with a CBR (Constant Bit Rate) traffic source generating packets of 50 bytes each. Out of the total 400 nodes, $10 \%$ are selected randomly to send traffic to the coordinator

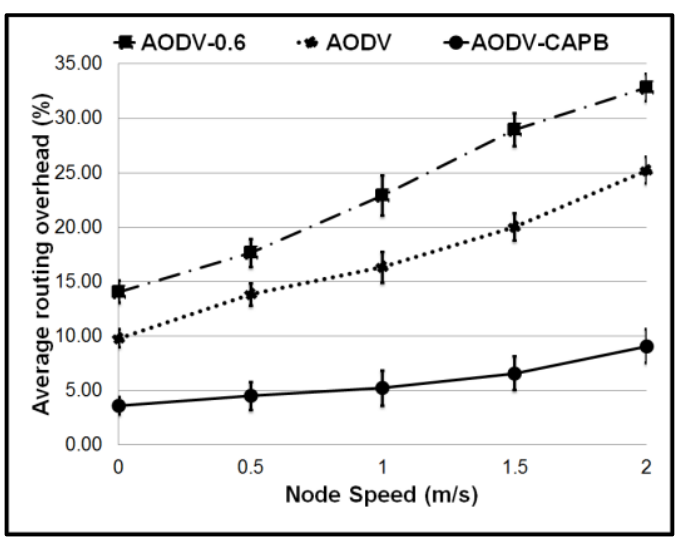

Fig. 2. Routing Overhead vs Node Speed

node. For energy consumption analysis, each node has an initial energy of 100 joules.

The scenario uses fixed number of nodes and node speed is varied. Simulation results are obtained by averaging the results of 30 runs within the same confidence interval of $95 \%$, each using a different seed value and lasting for 500 seconds. The seed value is used in the mobility model to yield different mobility profiles and to set the initial location of each node.

\section{B. Simulation results and analysis}

The performance of different broadcasting schemes in LRWSNs has been evaluated using four metrics namely routing overhead, throughput, end-to-end delay and average energy consumption.

\section{Routing overhead}

Routing overhead is defined as the ratio of the number of routing packets (control packets) transmitted per data packet received. Figure 2 shows the average routing overhead as a function of node speed.

Since the network topology is more dynamic due to increased node speed, routes get expired quickly and nodes trigger new route discovery more often to replace the expired routes. This leads to higher routing overhead for all schemes as the node speed increases.

Fixed probabilistic scheme (AODV-0.6) reduces the number of RREQ blindly which often affects the RREQ packets reachability to the destination node negatively and the node has to trigger a route discovery mechanism more often leading to higher overhead. Pure AODV scheme is slightly better than AODV-06 because it achieves better RREQ reachability to the destination and it triggers less frequent route discovery.

The CAPB scheme achieves significantly lower overhead as compared to the other two schemes. This is because CAPB avoids unnecessary rebroadcasts of RREQ packets and adapts rebroadcast probability to the node density and SINR ratio dynamically. 


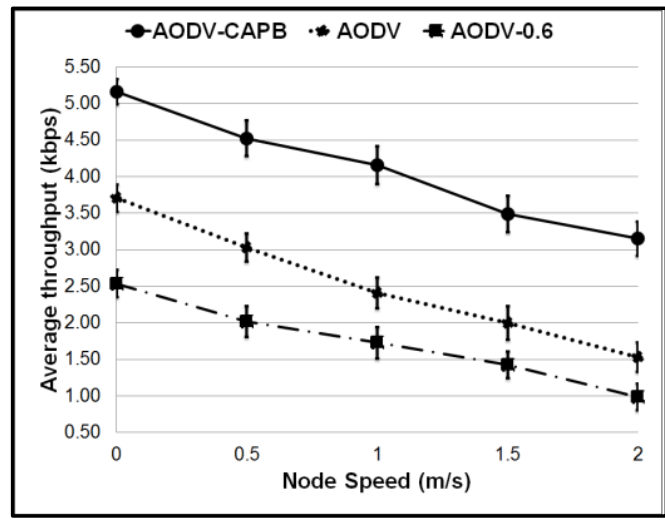

Fig. 3. Average throughput vs. Node Speed

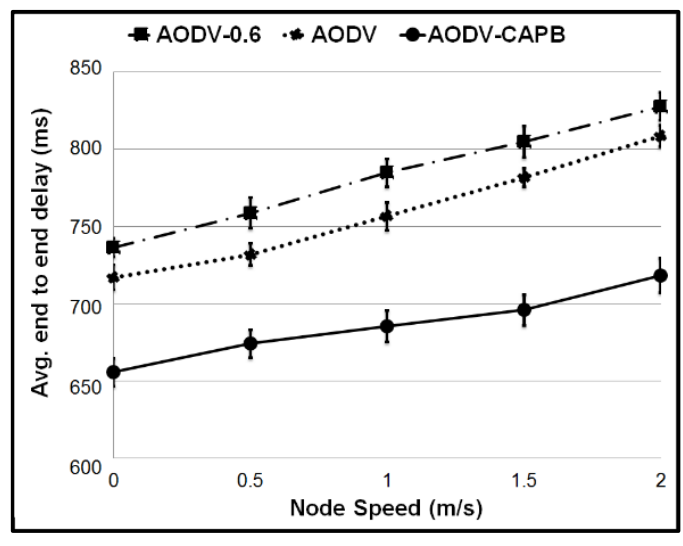

Fig. 4. Average end to end delay vs. Node Speed

\section{Average throughput}

Throughput is defined as the amount of data received by a node per unit of time. Figure 3 shows the throughput, calculated at the application layer, averaged over all source nodes as a function of node speed.

In general, increasing node speed changes the neighbouring nodes and network topology which causes a temporary pause in data transmission until the new route is established. Consequently, the average throughput falls with increasing node speed because routes are broken more often. The more time it needs to re-establish the route, the less the through would be. Inefficient and blind decisions on rebroadcasting the RREQ packet causes extra delay and penalises the throughput.

The CAPB achieves throughput significantly higher than the other two schemes. Better informed and adaptive rebroadcasting decision in CAPB scheme helps to increase the RREQ reachability to the destination node while keeping the routing overhead at the minimum which leads to higher throughput.

\section{Average end-to-end delay}

The average end-to-end delay is the average time a packet takes to reach from the source node to the destination node (PAN coordinator in this case). It includes all delays caused by buffering during route discovery, queuing at the interface queue, retransmission delays at the MAC, propagation delay and transmission delay. Figure 4 presents the average end to end delay for all nodes as a function of node speed. With the increase

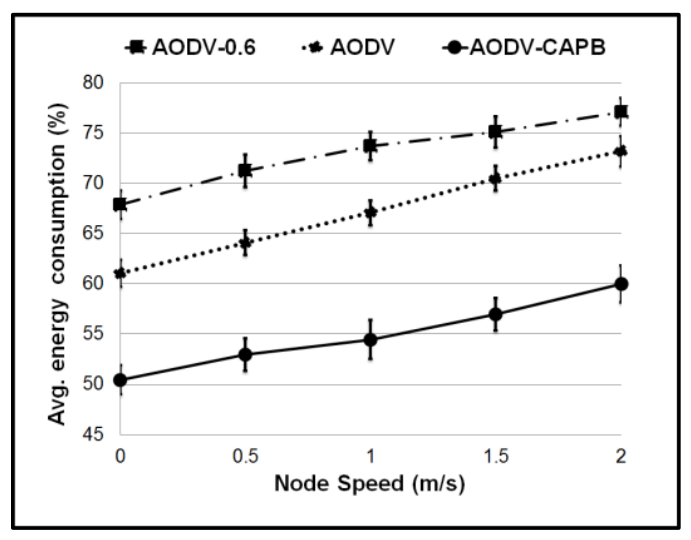

Fig. 5. Energy Consumption vs. Node Speed

in speed, the average end-to-end delay increases. It is because route breaking and rebuilding happen more often as the speed increases.

The CAPB average end-to-end delay is better than the other schemes. It is because the CAPB produces less routing traffic, which reduces the contention and collision, and it enhances the reachability of RREQ packets to the destination. This helps to rebuild the routes faster than other two schemes.

\section{Average energy consumption}

Energy consumption accounts for the energy consumed in transmitting, forwarding and receiving packets (both data and routing packets). Figure 5 depicts the energy consumption averaged over all nodes for different node speeds. The CAPB average energy consumption is much lower than the other two schemes. The CAPB energy saving is achieved by adapting the RREQ rebroadcasting to current channel conditions and neighbouring node density. This helps to reduce unnecessary transmissions of RREQ packet.

\section{CONCLUSION}

LR-WSN nodes are meant to deliver sensed data to the sink node reliably in ZigBee networks. Zigbee networks are characterised by their lower cost and lower power consumption. However, the default routing protocol in ZigBee networks is AODV that uses pure flooding to broadcast route discovery packet. This may lead to the broadcast storm problem and reduce the efficiency of ZigBee networks. This paper proposes and evaluates a Channel Adaptive Probabilistic Broadcast (CAPB) scheme that adapts the rebroadcast probability to the thermal noise, co-channel interference and node density in the neighbourhood dynamically. Based on extensive ns-2 simulations, the paper concludes that the CAPB scheme enhances the performance of ZigBee network compared to the standard AODV and the fixed probabilistic scheme significantly in terms of routing overhead, throughput, end-to-end delay and energy consumption.

\section{REFERENCES}

IEEE802.15.4, "Part 15.4: Wireless Medium Access Control (MAC) and Physical Layer (PHY) Specifications for Low-Rate Wireless Personal Area Networks (WPANs), 2006.

[2] M. Chen, T. Kwon, S. Mao, Y. Yuan, and V. C.M.Leung, "Reliable 
and energy efficient routing protocol in dense wireless sensor networks," Int. J. Sens. Netw., vol. 4, no. 1/2, pp. 104-117, 2008

[3] ZigBee, "ZigBee." [Online]. Available: http://www.zigbee.org/. [Accessed: 01-May-2016].

[4] P. Baronti, P. Pillai, V. W. C. Chook, S. Chessa, A. Gotta, and Y. F. $\mathrm{Hu}$, "Wireless sensor networks : A survey on the state of the art and the 802 . 15 . 4 and ZigBee standards," Comput. Commun., vol. 30, pp. 1655-1695, 2007.

[5] J. Yick, B. Mukherjee, and D. Ghosal, "Wireless sensor network survey," Comput. Networks, vol. 52, pp. 2292-2330, 2008.

[6] S.-J. Lee, E. M. Belding-Royer, and C. E. Perkins, "Ad hoc ondemand distance-vector routing scalability," ACM SIGMOBILE Mob. Comput. Commun. Rev., vol. 6, no. 3, p. 94, Jun. 2002.

[7] H. Y. Adarbah, S. Ahmad, B. Arafeh, and A. Duffy, "Efficient Broadcasting for Route Discovery in Mobile Ad-hoc Networks," in Proceedings of the International Symposium on Performance Evaluation of Computer and Telecommunication Systems, 2015, pp. $1-7$.

[8] "Wireless Medium Access Control (MAC) and Physical Layer (PHY) Specifications for Low Rate Wireless Personal Area Networks (WPANs).'[Online].Available:

https://standards.ieee.org/about/get/802/802.15.html. [Accessed: 01May-2016].

[9] M. Kasraoui, A. Cabani, and J. Mouzna, "IMPROVEMENT OF ZIGBEE ROUTING," in 2012 IEEE International Conference on Green Computing and Communications, Conference on Internet of
Things, and Conference on Cyber, Physical and Social Computing, 2012, pp. 788-793.

[10] B. Nefzi and Y. Song, "Performance Analysis and improvement of ZigBee routing protocol," in 7th IFAC International Conference on Fieldbuses \& Networks in Industrial \& Embedded Systems, 2007, pp. 199-206.

[11] F. Cuomo, S. Della Luna, U. Manco, and T. Melodia, "Routing in ZigBee : benefits from exploiting the IEEE 802 . 15 . 4 association tree," in IEEE International Conference on Communications, 2007. ICC '07, 2007, pp. 3271-3276.

[12] A. M. Ortiz, F. Royo, T. Olivares, and L. O. Barbosa, "Intelligent Route Discovery for ZigBee Mesh Networks," in 2011 IEEE International Symposium on a World of Wireless, Mobile and Multimedia Networks (WoWMoM), 2011, pp. 1-6.

[13] Y. Peng, Y. Li, Z. Lu, and J. Yu, "Method for Saving Energy in Zigbee Network," in 2009 5th International Conference on Wireless Communications, Networking and Mobile Computing, 2009, pp. 1-3.

[14] DEI, "NS2 Library: dei80211mr," 2014. [Online]. Available: http://www.isi.edu/nsnam/ns/doc/node193.html. [Accessed: 17-Feb2014].

[15] G. Lin, G. Noubir, and R. Rajamaran, "Mobility models for ad hoc network simulation," in Proceedings of 23rd Conference of the IEEE Communications Society (INFOCOM 2003), 2004, pp. 454-463. 